\title{
Thoracic surgeons' insights: Improving thoracic surgery outcomes during the Coronavirus Disease 2019 pandemic
}

Ke-Neng Chen, MD, ${ }^{\mathrm{a}}$ Shugeng Gao, MD, ${ }^{\mathrm{b}}$

Lunxu Liu, MD, ${ }^{\mathrm{c}}$ Jianxing He, MD, ${ }^{\mathrm{d}}$

Ge-Ning Jiang, MD, ${ }^{\mathrm{e}}$ and Jie $\mathrm{He}, \mathrm{MD}^{\mathrm{b}}$

At present, Coronavirus Disease 2019 (COVID-19) is raging worldwide, and almost all thoracic surgeons are facing unprecedented challenges. In this special period, how can we treat patients with thoracic disease safely and, particularly, how can we perform thoracic surgery safely? In addition, how do we prevent COVID-19 infection in the hospital and protect medical personnel? Other questions we must answer are whether patients are more susceptible to COVID-19 after thoracic surgery and what are the clinical manifestations and outcomes of infected postoperative patients. Faced with the global public health issue of COVID-19, every medical worker is responsible and obligated to share experiences and lessons in a timely manner to help humans through the present difficulties. Therefore, we strongly support our colleagues from China in their effort to share their experiences and lessons from combating COVID-19.

Peng and colleagues ${ }^{1}$ performed a cohort study from the area with a global high incidence of COVID-19, which has important reference value for guiding the daily work of thoracic surgery in the current environment of COVID-19. Peng and colleagues ${ }^{1}$ reported their

\footnotetext{
From the a Department of Thoracic Surgery, Key Laboratory of Carcinogenesis and Translational Research (Ministry of Education), Peking University Cancer Hospital \& Institute, Beijing, China; 'bepartment of Thoracic Surgery, National Cancer Center/Cancer Hospital, Chinese Academy of Medical Science and Peking Union Medical College, Beijing, China; ${ }^{c}$ Department of Thoracic Surgery, West China Hospital, Sichuan University, National Key Discipline of Surgery (Thoracic and Cardiovascular Surgery), Chengdu, China; ${ }^{\mathrm{d}}$ Department of Thoracic Oncology and Surgery, China State Key Laboratory of Respiratory Disease and National Clinical Research Center for Respiratory Disease, The First Affiliated Hospital of Guangzhou Medical University, Guangzhou, China; and ${ }^{\mathrm{e}}$ Department of Thoracic Surgery, Shanghai Pulmonary Hospital, Tongji, University School of Medicine, Shanghai, China.

This article has been co-published in The Journal of Thoracic and Cardiovascular Surgery, The Annals of Thoracic Surgery, and the European Journal of CardioThoracic Surgery.

Received for publication April 6, 2020; revisions received April 11, 2020; accepted for publication April 14, 2020; available ahead of print June 4, 2020.

Address for reprints: Jie He, MD, Department of Thoracic Surgery, National Cancer Center/Cancer Hospital, Chinese Academy of Medical Sciences, 17 Panjiayuan Nanli, Chaoyang District, Beijing 100021, China (E-mail: prof.hejie@263.net); or Ke-Neng Chen, MD, Department of Thoracic Surgery, Peking University Cancer Hospital, 52 Fucheng Rd, Haidian District, Beijing 100142, China (E-mail: chenkeneng@bjmu.edu.cn).

J Thorac Cardiovasc Surg 2020;160:597-600

$0022-5223 / \$ 36.00$

Copyright (c) 2020 by Jointly between The American Association for Thoracic Surgery, The Society of Thoracic Surgeons, and the European Association for CardioThoracic Surgery. Published by Elsevier Inc.

https://doi.org/10.1016/j.jtcvs.2020.04.038
}

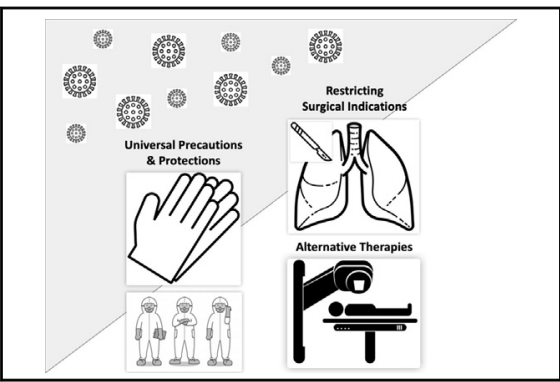

Limiting surgery and implementing alternative strategies to prevent COVID-19 infection.

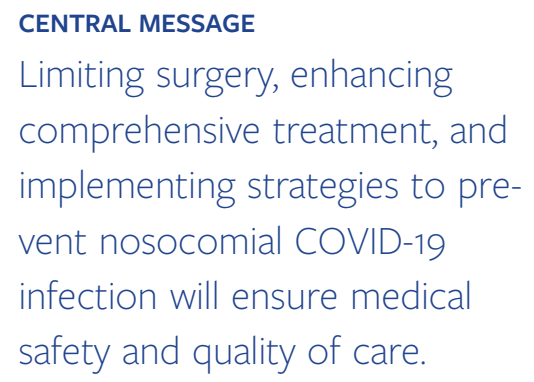

experience of 11 patients infected with COVID-19 after thoracic surgery in a high-volume center in China during the pandemic. There were 205 patients admitted, including 119 cases with surgery and 86 cases without surgery. After surgery, 11 patients and 8 medical workers were infected with COVID-19. ${ }^{2}$ The investigators analyzed the clinical symptoms, laboratory tests, imaging manifestations, and treatment outcomes of these patients. The mortality of infected patients after thoracic surgery was $27.3 \%$, which was 10 times higher than the usual $2 \%$ mortality $^{3}$ and higher than the $4.3 \%$ mortality of COVID-19-infected cases without surgery according to another report. ${ }^{4}$ On the basis of these data, we must adjust our daily work to meet the current challenges. Although the risk levels vary from region to region, and the measures taken are also different, it is difficult for one recommendation to cover the scope of all medical centers. Nevertheless, the recommendations we are proposing should have certain reference value and warning function to thoracic surgeons from different regions.

\section{UNIVERSAL PRECAUTION}

The World Health Organization has declared COVID-19 a global health concern and pandemic. Because the economic development, medical resources, and clinical practice of different regions are not the same, "universal 
precaution" could have a different context while the concept remains the same. This point is important; thus, we suggest that all patients who come to the hospital for surgery be treated under the "universal precaution" principle on the premise of enough supplies, particularly in the center of the epidemic. Studies also show that many asymptomatic patients are infectious, and their infectivity is no less than that of symptomatic patients. ${ }^{5}$ In addition, there is the possibility for sudden acceleration of infection. Therefore, a simple inquiry about fever, cough, and shortness of breath in the past 2 weeks and any history of recent wildlife contact cannot help us identify problematic patients. ${ }^{6}$ It is more reasonable to use universal precaution for COVID-19 screening of every new patient.

\section{RESTRICTING SURGICAL INDICATIONS}

Although Peng and colleagues ${ }^{1}$ did not provide detailed information about the specific diseases of the 119 surgical patients, evaluating ground-glass opacities (GGOs) seen on chest computed tomography (CT) scans has become an important part of clinical practice of some, perhaps many, thoracic surgery teams in China. Numerous studies have proved that the incidence of lung adenocarcinoma presented as GGO on CT is increasing recently. Some clinical features and biological behaviors of this kind of tumor are different from the traditional lung adenocarcinoma presented as solid nodules on imaging. For example, studies showed that GGO-like lung adenocarcinoma is more common in Asian and young women than in the traditional high-risk population with lung cancer, such as senior men and smokers. ${ }^{7-9}$ Different from conventional solid lung adenocarcinomas, this special type of lung adenocarcinoma, which emerged 20 years ago, exhibits indolent growth as its most obvious feature. ${ }^{10}$ Moreover, the pure GGO lung adenocarcinoma as screened by low-dose CT in an asymptomatic population grows even more slowly. In the 8th edition TNM staging system, it is classified as $\mathrm{c} 0$ stage. For semi-solid GGO, T is calculated on the basis of the diameter of the solid component of the tumor. ${ }^{11}$ In addition, data support the conclusion that the GGO component is a favorable prognostic factor for lung adenocarcinoma. ${ }^{8,9}$ Coincidentally, the early imaging manifestation of COVID-19 is also predominantly ground-glass shadow. ${ }^{1}$ Although there are some distinguishing features with GGO-like lung adenocarcinoma, it is still necessary to attach great importance to possible misdiagnosis. In view of the indolence and relatively good prognosis of GGO-like lung adenocarcinoma, in the current environment of COVID-19 pandemic, we call for the cessation of all thoracic surgeries for GGO-like lung adenocarcinoma with a diameter of less than $30 \mathrm{~mm}$ during the COVID-19 pandemic. ${ }^{12}$ Of course, GGO-like lung adenocarcinoma should also be differentiated from other benign diseases to avoid the risk of infection caused by unnecessary surgery, such as ground-glass shadow caused by hemoptysis in the lung, radiation-induced pneumonia, exogenous lipid pneumonia, and immunotherapy-related pneumonitis.

\section{ENHANCING PREOPERATIVE INDUCTION TREATMENT AND INITIATING ALTERNATIVE TREATMENT}

According to the survey of Rocco and colleagues ${ }^{13}$ in Europe and North America, as well as the data of the ADJUVANT/CTONG1104 Study in China, ${ }^{14}$ there are large treatment strategy differences for IIIA-N2 locally advanced non-small cell lung cancer. Certainly, in patients in whom surgery may be indicated for IIIA-N2, surgery should follow preoperative chemotherapy for oncologic reasons ${ }^{15}$ and to delay the surgery beyond the window of the pandemic. Patients who might be considered marginal candidates for surgery might be treated with chemoradiation followed by immunotherapy, according to the PACIFIC trial, ${ }^{16}$ avoiding the risks of hospitalization during the pandemic without compromising treatment for cure. There was no statistically significant difference in overall survival between chemoradiotherapy alone versus chemoradiotherapy plus surgery. ${ }^{17}$ As well, during the COVID-19 pandemic, clinicians should rely to a greater extent on positron emission tomography for staging $^{18}$ to replace invasive biopsies and reduce the chance of infection among medical workers.

Conversely, esophageal cancer is an important disease in the clinical practice of thoracic surgery, and esophagectomy is the earliest and most important treatment method so far. In China, squamous cell carcinoma accounts for $90 \%$ of esophageal cancer, ${ }^{19}$ and $90 \%$ of these cancers show locally advanced disease at the first visit. There is strong clinical evidence that the long-term survival of patients with locally advanced esophageal cancer is significantly better with induction chemoradiation followed by surgery compared with up-front surgery. ${ }^{20,21}$ However, in clinical practice in China, preoperative treatment of locally advanced esophageal cancer is not universal because of different understanding of multimodality treatments, a lack of multidisciplinary cooperation, and unbalanced deployment of radiation technology and equipment. Thus, postoperative adjuvant treatment accounts for more than $50 \%$ of the comprehensive treatment for esophageal cancer, ${ }^{22}$ which not only affects the long-term survival of patients but also places patients at risk of infection during the COVID-19 pandemic. Preoperative induction chemoradiotherapy improves the curative effect of surgery, and some scholars even question the role of surgery in comprehensive treatment of esophageal squamous cell carcinoma. These specialists concluded that overall survival after concurrent chemoradiation was not statistically different from survival after trimodality therapy; therefore, concurrent chemoradiation could replace surgery entirely. ${ }^{23}$ Although this viewpoint is controversial because of a lack of strong evidence, under the current COVID-19 pandemic situation, 
we should more strictly consider the indications for surgery. We should choose more conservative treatments for seniors, those with severe comorbidities, and those with advanced disease to decrease the risks associated with surgery under this special situation. Conversely, we have noticed that preoperative radiation dosage is a controversial issue in preoperative chemoradiation. In fact, the "curative" radiation dosage in Asian countries is similar to the "preoperative induction" radiation dosage in western countries. In this special period, we might as well give "curative" concurrent chemoradiotherapy (radiation dose similar to the Western preoperative dose) and consider surgery in a "salvage" situation after close observation and follow-up.

\section{PROTECTION PROTOCOLS DURING EMERGENCY OPERATIONS FOR PATIENTS WITH CONFIRMED OR SUSPECTED COVID-19 INFECTION}

We can and should try to avoid elective operations during the outbreak. However, we cannot avoid emergency surgery, even for individuals with confirmed or suspected COVID-19 infection. In these circumstances, all medical workers participating in the care of patients with confirmed or suspected COVID-19 infection need 3-level protection, including medical masks, protective clothing, goggles/protective masks, double gloves, shoe covers, and so forth. All staff who participated in the care of these patients should undergo medical observation for 14 days after surgery. Patients undergoing emergency surgery should remain isolated from the preoperative preparation stage to postoperative treatment, and they must wear masks at all time. For operations in the central areas with continuous outbreak of the epidemic, even for patients without confirmed or suspected infection, reverse transcription polymerase chain reaction of samples taken from upper nasopharyngeal swabs should be performed, and preoperative preparations should be made immediately before the operations. If the test result is negative, it is recommended that patients remain in an isolation ward, and a 3-level protection protocol should be adopted during the operations. For patients with a negative test result before surgery, after intubation, the lower respiratory tract secretions should be tested again.

Surgery shall be performed in an independent negative pressure operating room, and the number of participants shall be limited. After entering the operating room, no one shall enter or leave arbitrarily. All supplies shall be in the charge of nurses outside the operating room. Intubation should be performed after anesthesia. A disposable filter should be arranged between the tracheal intubation and the respiratory tract to reduce contamination of the respiratory tract. More attention should be devoted to protection when suctioning sputum, and suction should be performed in a closed environment. During the operations, the use of energy devices and energy power should be reduced to limit aerosol caused by the devices. For examination, surgical specimens should be sealed in a double-layered bag. After surgery, the operating room should be disinfected thoroughly, and it can be reused only after passing a sampling test by the infection management department.

Some patients undergoing elective surgery, but could not wait for too long because of the disease condition, should be observed in a transitional ward for 2 weeks. Two weeks before surgery, patients should have a chest CT for COVID-19 screening and routine blood assay, C-reactive protein, procalcitonin, and influenza $\mathrm{A}$ and $\mathrm{B}$ examination within 1 week. If infection is suspected, a further upper nasopharyngeal swab test for COVID-19 should be performed. In fact, all patients should undergo a swab test before surgery.

Postoperative fever, shortness of breath, and cough are common clinical manifestations for surgical patients. Fever can be caused by postoperative absorption and bacterial pneumonia of the respiratory tract or pleural infection. Postoperative chest tightness and shortness of breath are also common, due to bacterial pneumonia, atelectasis, and acute respiratory distress syndrome. Rare causes include pulmonary embolism, pulmonary volvulus, interstitial pneumonia, and bronchopleural fistula. These cases should be identified carefully and differentiated in a timely manner. In addition, because of symptom overlap with COVID-19 infection, these conditions should be differentiated from COVID-19.

In the context of the current COVID-19 pandemic, thoracic surgeons should avoid surgery for benign tumors. Even for malignancies, strict control of the indications for operations is recommended, comprehensive treatment should be emphasized, and alternative treatment strategies should be implemented to avoid COVID-19 infection in hospital and ensure medical safety and quality.

\section{Conflict of Interest Statement}

The authors reported no conflicts of interest.

The Journal policy requires editors and reviewers to disclose conflicts of interest and to decline handling or reviewing manuscripts for which they may have a conflict of interest. The editors and reviewers of this article have no conflicts of interest.

\section{References}

1. Peng S, Huang L, Zhao B, Zhou S, Braithwaite I, Zhang N, et al. Clinical course of coronavirus disease 2019 in 11 patients after thoracic surgery and challenges in diagnosis. J Thorac Cardiovasc Surg. 2020;160:585-92.e2.

2. Li YK, Peng S, Li LQ, Wang Q, Ping W, Zhang N, et al. Clinical and transmission characteristics of Covid-19-a retrospective study of 25 cases from a single thoracic surgery department. Curr Med Sci. 2020;40:295-300.

3. Allen MS, Darling GE, Pechet TT, Mitchell JD, Herndon JE II, Landreneau RJ, et al. Morbidity and mortality of major pulmonary resections in patients with early-stage lung cancer: initial results of the randomized, prospective ACOSOG Z0030 trial. Ann Thorac Surg. 2006;81:1013-20. 
4. Wang D, Hu B, Hu C, Zhu F, Liu X, Zhang J, et al. Clinical characteristics of 138 hospitalized patients with 2019 novel coronavirus-infected pneumonia in Wuhan, China. JAMA. 2020;323:1061-9.

5. Bai Y, Yao L, Wei T, Tian F, Jin DY, Chen L, et al. Presumed asymptomatic carrier transmission of COVID-19. JAMA. 2020;323:1406-7.

6. Qiu J. Covert coronavirus infections could be seeding new outbreaks. Nature. March 20, 2020 [Epub ahead of print].

7. Zhang Y, Jheon S, Li H, et al. Results of low-dose computed tomography as a regular health examination among Chinese hospital employees. J Thorac Cardiovasc Surg. November 14, 2019 [Epub ahead of print].

8. Chen KN. Commentary: pay attention to low-risk populations for lung cancer, but cautiously interpret ground-glass nodules screened by low-dose computed tomography scan. J Thorac Cardiovasc Surg. November 27, 2019 [Epub ahead of print].

9. Chen KN. The diagnosis and treatment of lung cancer presented as ground-glass nodule. Gen Thorac Cardiovasc Surg. December 10, 2019 [Epub ahead of print].

10. Detterbeck FC. Achieving clarity about lung cancer and opacities. Chest. 2017; 151:252-4.

11. Travis WD, Asamura H, Bankier AA, Beasley MB, Detterbeck F, Flieder DB, et al. The IASLC lung cancer staging project: proposals for coding T categories for subsolid nodules and assessment of tumor size in part-solid tumors in the forthcoming Eighth Edition of the TNM classification of lung cancer. $J$ Thorac Oncol. 2016;11:1204-23.

12. Suzuki S, Sakurai H, Yotsukura M, Masai K, Asakura K, Nakagawa K, et al. Clinical features of ground glass opacity-dominant lung cancer exceeding 3.0 $\mathrm{cm}$ in the whole tumor size. Ann Thorac Surg. 2018;105:1499-506.

13. Rocco G, Nason K, Brunelli A, Varela G, Waddell T, Jones DR. Management of stage IIIA (N2) non-small cell lung cancer: a transatlantic perspective. J Thorac Cardiovasc Surg. 2016;151:1235-8.

14. Zhong WZ, Wang Q, Mao WM, Xu ST, Wu L, Shen Y, et al. Gefitinib versus vinorelbine plus cisplatin as adjuvant treatment for stage II-IIIA (N1-N2) EGFRmutant NSCLC (ADJUVANT/CTONG1104): a randomised, open-label, phase 3 study. Lancet Oncol. 2018;19:139-48.
15. Martins RG, D’Amico TA, Loo BW Jr, Pinder-Schenck M, Borghaei H, Chaft JE, et al. The management of patients with stage IIIA non-small cell lung cancer with N2 mediastinal node involvement. J Natl Compr Canc Netw. 2012;10:599-613.

16. Antonia SJ, Villegas A, Daniel D, Vicente D, Murakami S, Hui R, et al. Durvalumab after chemoradiotherapy in stage III non-small-cell lung cancer. $N$ Engl J Med. 2017:377:1919-29.

17. Albain KS, Swann RS, Rusch VW, Turrisi AT 3rd, Shepherd FA, Smith C, et al. Radiotherapy plus chemotherapy with or without surgical resection for stage III non-small-cell lung cancer: a phase III randomised controlled trial. Lancet. 2009; 374:379-86.

18. Schmidt-Hansen M, Baldwin DR, Hasler E, Zamora J, Abraira V, Roqué I Figuls M. PET-CT for assessing mediastinal lymph node involvement in patients with suspected resectable non-small cell lung cancer. Cochrane Database Syst Rev. 2014;2014:CD009519.

19. Mao YS, Gao SG, Wang Q, Shi XT, Li Y, Gao WJ, et al. Analysis of a registry database for esophageal cancer from high-volume centers in China. Dis Esophagus. December 20, 2019 [Epub ahead of print].

20. Shapiro J, van Lanschot JJB, Hulshof MCCM, van Hagen P, van Berge Henegouwen MI, Wijnhoven BPL, et al. Neoadjuvant chemoradiotherapy plus surgery versus surgery alone for oesophageal or junctional cancer (CROSS): long-term results of a randomised controlled trial. Lancet Oncol. 2015;16: 1090-8.

21. Yang H, Liu H, Chen Y, Zhu C, Fang W, Yu Z, et al. Neoadjuvant chemoradiotherapy followed by surgery versus surgery alone for locally advanced squamous cell carcinoma of the esophagus (NEOCRTEC5010): a phase III multicenter, randomized, open-label clinical trial. J Clin Oncol. 2018;36: 2796-803.

22. Chen K-N. Commentary: esophageal squamous cell carcinoma: a disease requiring more research. J Thorac Cardiovasc Surg. 2020;160:553-4.

23. Bedenne L, Michel P, Bouché O, Milan C, Mariette C, Conroy T, et al. Chemoradiation followed by surgery compared with chemoradiation alone in squamous cancer of the esophagus: FFCD 9102. J Clin Oncol. 2007;25:1160-8. 